\title{
Array antenna pattern synthesis for fixed amplitude and limited phase range using genetic algorithm
}

\author{
Hiroshi Hashiguchi $^{1, \text { a) }}{ }^{\text {, Naobumi Michishita }}{ }^{1}$, Hisashi Morishita ${ }^{1}$, \\ and Hiroyuki Arai ${ }^{2}$ \\ ${ }^{1}$ Graduate School of Science and Engineering, National Defense Academy \\ 1-10-20 Hashirimizu, Yokosuka, Kanagawa, 239-8686, Japan \\ ${ }^{2}$ Graduate School of Engineering, Yokohama National University \\ 79-5 Tokiwadai, Hodogaya, Yokohama, Kanagawa, 240-8501, Japan \\ a)hhiroshi@nda.ac.jp
}

Abstract: This paper presents array antenna pattern synthesis with a fixed amplitude and limited phase range using genetic algorithm (GA). The array antenna can synthesize its pattern by controlling the amplitude and phase of each antenna element. However, a large phase shifter is required to control an arbitrary phase range of $360^{\circ}$. This proposed method can synthesize a cosecant squared beam (CSB) in the case of a 16-element, half-wavelength element spacing linear array with a fixed amplitude and limited phase range of less than $180^{\circ}$.

Keywords: array antenna, cosecant squared bean, array pattern synthesis, genetic algorithm

Classification: Antennas and Propagation

\section{References}

[1] H. Lebret and S. Boyd, "Antenna array pattern synthesis via convex optimization," IEEE Trans. Signal Process., vol. 45, no. 3, pp. 526-532, March 2015. DOI: $10.1109 / 78.558465$

[2] T. Chiba, "On a pattern synthesis method for a linear array," IEEE International Convention Record, part 5, pp. 172-179, March 1966. DOI: 10.1109/irecon. 1966.1147709

[3] B. Sun, C. Liu, Y. Liu, X. Wu, Y. Li, and X. Wang, "Conformal array pattern synthesis and activated elements selection strategy based on PSOGSA algorithm," Int. J. Antennas Propag., vol. 2015, 2015. DOI: 10.1155/2015/858357

[4] E.H. Kenane, F. Djahli, and A. Bartil, "Synthesis of cosecant linear antenna array pattern using a novel modified invasive weeds optimization," Elektronika Ir Elektrotechnika, vol. 21, no. 5, pp. 86-89. Oct. 2015. DOI: 10.5755/j01.eie.21.5.13332

[5] P. Kumar and A. Singhm, "Phase only pattern synthesis for antenna array using genetic algorithm for radar application," Indian Journal of Radio and Space Physics, vol. 42, no. 1, pp. 259-264.

[6] M. Yamamoto, H. Arai, Y. Ebine, and M. Nasuno, "Simple design method for cosec square with null-filling in equispaced linear Array," IEICE Trans. Commun. 
(Japanese Edition), vol. J101-B, no. 8, pp. 619-626, Aug. 2018.

[7] C. Park, H. Kim, J. Kim, and K. Min, "Compact phase shifter for 4G base station antenna," International Symposium on Antenna and Propagation (ISAP 2018), Busan, Korea, pp. 333-334, FrA1-1, Oct. 2018.

[8] M.J. Buckley, "Synthesis of shaped beam antenna patterns using implicity constrained current elements," IEEE Trans. Antenna Propag., vol. 44, no. 2, pp. 192197, Feb. 1996. DOI: 10.1109/8.481647

[9] B. Lindmark, "Analysis of pattern null-fill in linear arrays," 2013EuCAP, pp. 1457-1461, April 2013.

\section{Introduction}

Array antennas are used in many applications, such as base stations, and the desired array pattern can be synthesized by controlling the amplitude and phase, called weight [1]. Several numerical methods have been proposed to calculate these amplitudes and phases, such as Fourier series expansion [2] and others [3, 4]. Because many amplifiers are required to control the amplitude, resulting in low power efficiency, the GA and null-filling methods have been proposed as array antenna pattern synthesis methods with fixed amplitudes [5,6]. If the array antenna pattern can be synthesized with a limited phase range, phase shifters that use a slider for phase shifting, such as in [7], can be downsized. However, to the best of our knowledge, array antenna pattern synthesis with a fixed amplitude and limited phase range has not been proposed. In this paper, we propose an array antenna pattern synthesis with a fixed amplitude and limited phase range using GA. This paper is organized as follows: First, we consider the optimization procedure for the array antenna pattern synthesis using GA owing to many parameters that should be optimized. Second, we show the results of the optimization procedure.

\section{Optimization procedure}

Before the array pattern synthesis using the GA, we consider the optimization procedure owing to many parameters that should be optimized. We categorize the parameters into the desired array pattern, the parameters of the GA, the evaluation function, and the parameters of the array antenna, respectively, as shown in Table 1. As the desired array pattern, a cosecant squared beam (CSB) is employed because there is a report that compares the phase range [6]. In addition, the range of the CSB is set from $0^{\circ}$ to $30^{\circ}$. GA can optimize parameters through selection, crossover, and mutation. We used that the crossover is uniform, the mutation is bit string, and the selection is roulette wheel selection. The parameters of the GA are categorized into population, number of generations, crossover probability, and mutation probability. We tentatively set the parameters of the array antenna to investigate the GA parameters and compare the values of the following evaluation functions:

$$
E=\sum_{\theta=-90}^{90}(D(\theta)-G(\theta)) W(\theta)
$$


Table I. Parameters for optimization

\begin{tabular}{|l|l|c|c|}
\hline Category & Name & Symbol & Type or Value \\
\hline Desired array pattern & Cosecant squared beam & - & - \\
\hline \multirow{4}{*}{ GA } & Population & - & 300 \\
\cline { 2 - 4 } & Number of generation & - & 30 \\
\cline { 2 - 4 } & Crossover probability & - & $90 \%$ \\
\cline { 2 - 4 } & Mutation probability & - & $20 \%$ \\
\hline Evaluation & unit & - & linear \\
\cline { 2 - 4 } & weight (SL region) & $w_{1}$ & 0.05 \\
\cline { 2 - 4 } & weight (CSB region) & $w_{2}$ & 1 \\
\cline { 2 - 4 } & Sidelobe level & $S$ & 0.01 \\
\hline Array antenna & Number of element & $N$ & 16 \\
\cline { 2 - 4 } & Element spacing & $d$ & $0.5 \lambda$ \\
\cline { 2 - 4 } & Amplitude distribution & $A_{n}$ & cosine, cosine square \\
\cline { 2 - 4 } & Phase range & $\phi_{n}$ & less than 180 deg. \\
\hline
\end{tabular}

where $D(\theta)$ is the desired array pattern (pattern mask) and $W(\theta)$ is the weight function, which comprises the weight coefficient as follows:

$$
D(\theta)=\left\{\begin{array}{l}
S\left(-90^{\circ}<\theta<0^{\circ}\right) \\
\csc ^{2}\left(\theta=5^{\circ}\right)\left(0^{\circ}<\theta<5^{\circ}\right) \\
\csc ^{2}\left(5^{\circ}<\theta<30^{\circ}\right) \\
S\left(30^{\circ}<\theta<90^{\circ}\right)
\end{array}\right.
$$

where $S$ is the sidelobe level of the desired pattern. Because $\csc ^{2}\left(\theta=0^{\circ}\right)$, approaches infinity, we set $\theta$ of $5^{\circ}$, for maximum CSB from $0^{\circ}$ to $5^{\circ}$.

$$
W(\theta)=\left\{\begin{array}{l}
w_{1}\left(-90^{\circ}<\theta<0^{\circ}\right) \\
w_{2}\left(0^{\circ}<\theta<30^{\circ}\right) \\
w_{1}\left(30^{\circ}<\theta<90^{\circ}\right)
\end{array}\right.
$$

$G(\theta)$ is the array factor presented as follows:

$$
G(\theta)=\sum_{n=1}^{N} A_{n} \exp \left(-j k_{0} n d \sin \theta\right) \exp \left(-j \phi_{n}\right)
$$

where $N$ is the element number, $d$ is the element spacing, $A_{n}$ and $\phi_{n}$ are the amplitude and phase of each element, respectively, and $k_{0}$ is the wavenumber of $2 \pi / \lambda$. In the proposed method, the population generated by the GA is converted to phase $\phi_{n}$ in a limited phase range. Note that the $\mathrm{dB}$ or linear units of $\mathrm{D}(\theta)$ and $\mathrm{G}(\theta)$ affect the results of the array antenna pattern synthesis. The weighting coefficients of the evaluation function are divided into a CSB and sidelobe regions, where $w_{1}$ and $w_{2}$ are the sidelobe and CSB regions, respectively. The parameters of the array antenna are the number of elements $N$, element spacing $d$, amplitude $A_{n}$, and phase $\phi_{n}$. The number of elements $N$ equals 16, owing to the tournament feeding circuit, and the element spacing $d$ is $0.5 \lambda$ for a compact array antenna. Amplitude $A_{n}$, cosine distribution, and a cosine square distribution are investigated because these amplitudes are often used for array antennas. Based on ref $[6,8,9]$, CSB is synthesized with a phase range of $360^{\circ}$; thus, we aim to limit the phase range to $180^{\circ}$, which is half of phase range. 


\section{Results obtained using the optimization procedure}

To optimize the parameters of the GA, we tentatively set the amplitude as cosine and the phase range of $180^{\circ}$ for the array antenna parameters. In addition, we set the unit as linear, $w_{1}$ as 0.05 , and $w_{2}$ as 1 for the weighting coefficients of the evaluation function. Consequently, there is no remarkable tendency owing to the variation of the GA parameters; we set the population to 300 , the number of generations to 30 , the crossover of $90 \%$, and the mutation of $20 \%$. The unit of the evaluation function is linear because the array pattern of the CSB is stably obtained. Moreover, we investigate the weighting coefficients $w_{1}$ and $w_{2}$ and confirm that stable results are obtained when the weighting coefficient of the sidelobe region was small. Thus, the weighting coefficients of $w_{1}$ and $w_{2}$ are 0.05 and 1 , respectively. The parameter $S$ is 0.01 , because the array pattern of CSB and low sidelobe level is stably obtained. Figure. 1 shows the relationship between the array antenna pattern with a limited phase range in each amplitude case. The amplitude is fixed, as shown in Fig. 1(a), and the phase is optimized by the GA in a limited phase range to synthesize the desired array pattern. As shown in Fig. 1(b), the desired and synthesized array patterns show good agreement, however, the amplitude of the cosine distribution is slightly higher than the sidelobe level. Thus, the cosine-square distribution is used for the amplitude.

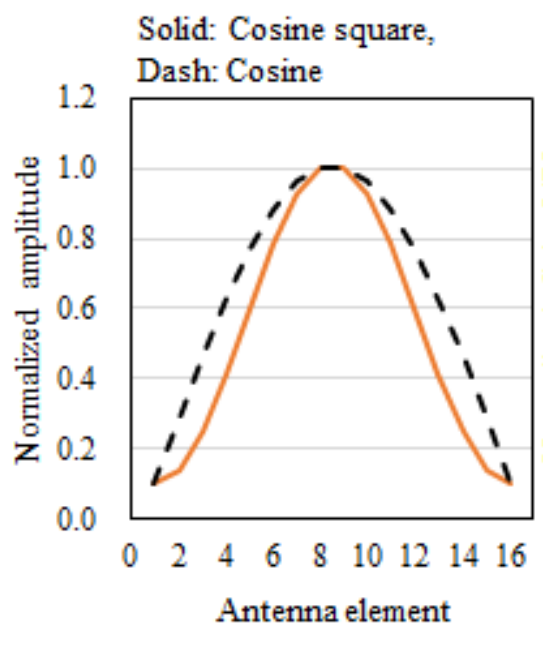

(a)
Solid: Cosine square, Dotted Dash: Cosine, Dash: Desired pattern

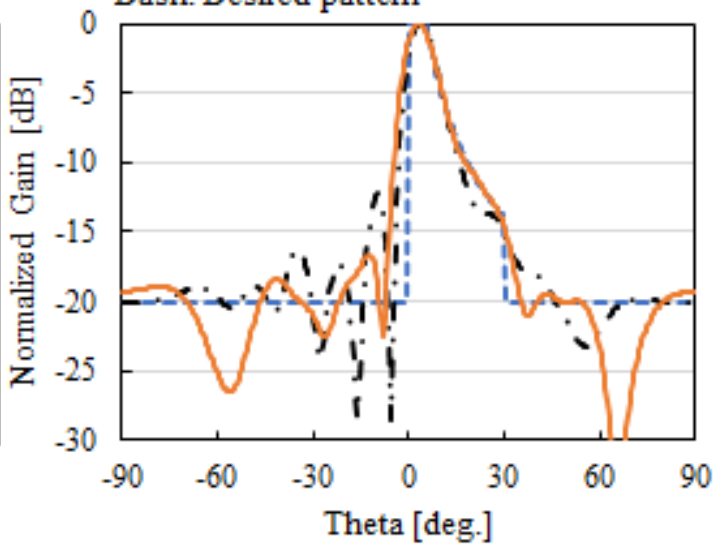

(b)

Fig. 1. Relation between phase only array pattern and amplitude; (a) normalized amplitude, (b) synthesized array pattern.

Finally, we synthesize an array antenna pattern with a more limited phase range of $180^{\circ}$ as shown in Fig. 2. Figure 2(a) shows the optimized phase range of the GA for the case of $140^{\circ}$ and $160^{\circ}$, respectively. Although the CSB region is slightly absent at $140^{\circ}$, a good CSB is synthesized in each case, as shown in Fig. 2(b). We cannot observe the CSB pattern when the limited phase range is less than $130^{\circ}$. The results show that CSB can be synthesized by the GA when the amplitude is cosine-squared, and the phase range is less than $140^{\circ}$. 


\section{Rect.: phase range $160^{\circ}$ Tri.: phase range $140^{\circ}$}

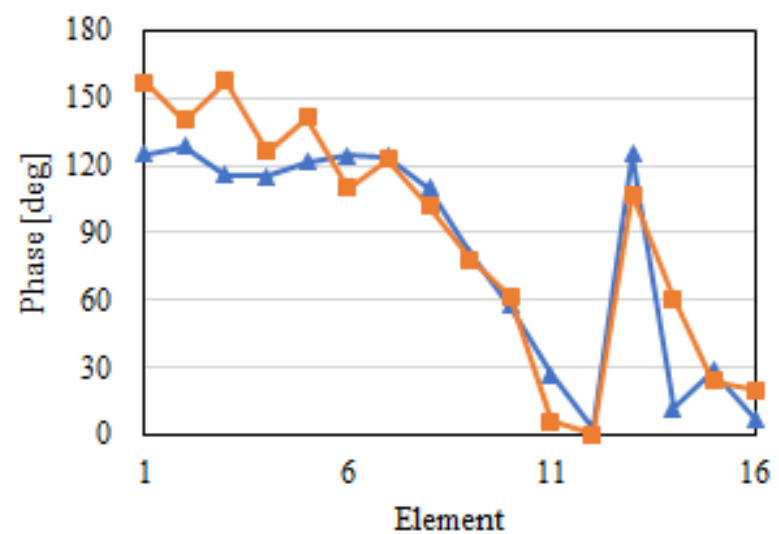

(a)

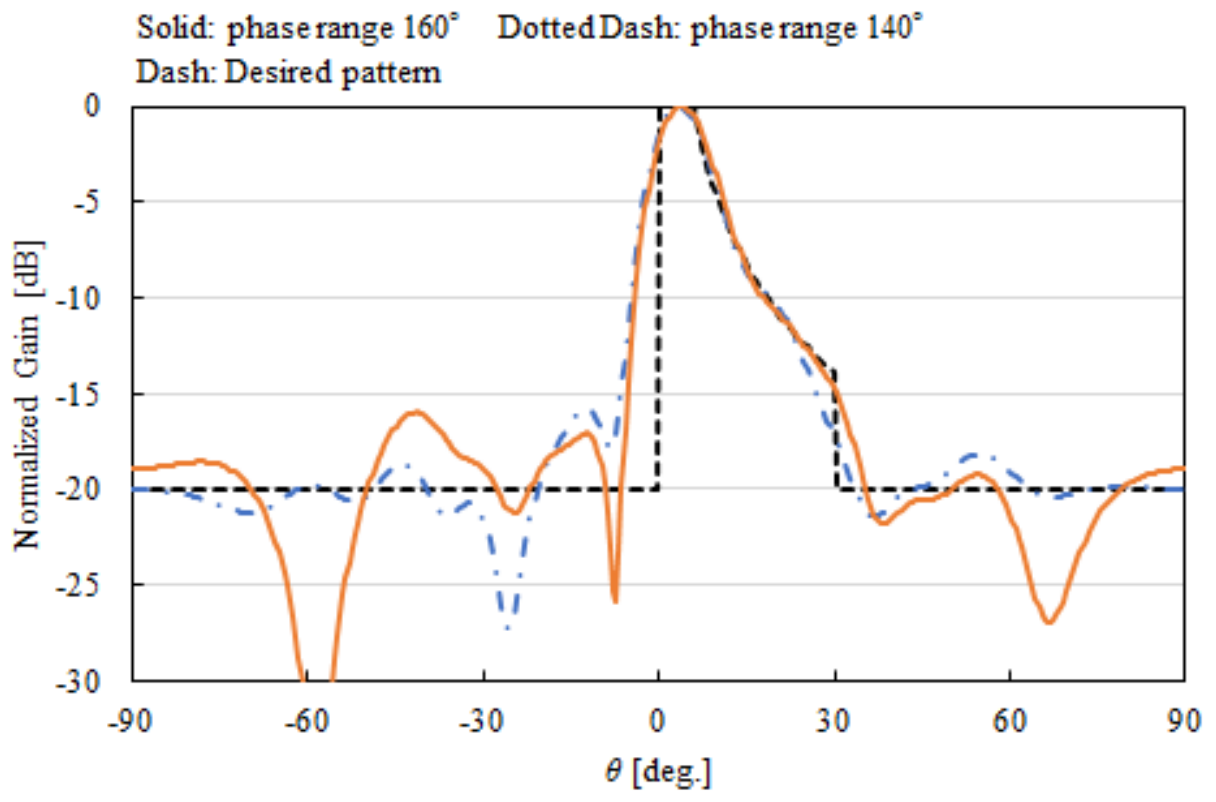

(b)

Fig. 2. Array antenna pattern synthesis by proposed method; (a) phase range, (c) synthesized pattern.

\section{Conclusion}

In this paper, we proposed an array pattern synthesis with a fixed amplitude and limited phase range using a GA. We confirmed that the proposed method can synthesize a CSB in the case of a 16-element, half-wavelength linear array with a cosine-squared amplitude of less than $140^{\circ}$. To demonstrate this method, designing antenna elements and phase shifters will be considered in future work. In addition, sidelobe level reduction will be considered in future work. 